-7. $@$ ERSpublications

Caution should be taken when adopting new ERS/ATS recommendation on 6-min walk test procedure http://ow.ly/PX9OB

Giancarlo Piaggi $^{1,2}$, Simone Gambazza ${ }^{2,3}$, Riccardo Guarise ${ }^{3}$ and Manuela Piran ${ }^{1}$

${ }^{1}$ IRCCS Salvatore Maugeri Foundation, Scientific Institute of Pavia, Division of Pulmonary rehabilitation, Pavia, Italy.

${ }^{2}$ Associazione Riabilitatori dell'Insufficienza Respiratoria (ARIR), Milan, Italy. ${ }^{3}$ Fondazione IRCCS Cà Granda Ospedale

Maggiore Policlinico, Cystic Fibrosis Centre, Milan, Italy.

Correspondence: Giancarlo Piaggi, IRCCS Salvatore Maugeri Foundation, Scientific Institute of Pavia, Division of Pulmonary Rehabilitation, Via Salvatore Maugeri 10, 27100 Pavia, Italy. E-mail: giancarlo.piaggi@fsm.it

Received: April 292015 | Accepted after revision: May 202015

Conflict of interest: None declared.

\title{
References
}

1 Singh SJ, Puhan MA, Andrianopoulos V, et al. An official systematic review of the European Respiratory Society/ American Thoracic Society: measurement properties of field walking tests in chronic respiratory disease. Eur Respir J 2014; 44: 1447-1478.

2 Holland AE, Spruit MA, Troosters T, et al. An official European Respiratory Society/American Thoracic Society technical standard: field walking tests in chronic respiratory disease. Eur Respir J 2014; 44: 1428-1446.

3 Jenkins S, Cecins N. Six-minute walk test: observed adverse events and oxygen desaturation in a large cohort of patients with chronic lung disease. Intern Med J 2011; 41: 416-22.

4 ATS Committee on Proficiency Standards for Clinical Pulmonary Function Laboratories. ATS statement: guidelines for the six-minute walk test. Am J Respir Crit Care Med 2002; 166: 111-117.

5 American Thoracic Society, American College of Chest Physicians. ATS/ACCP Statement on cardiopulmonary exercise testing. Am J Respir Crit Care Med 2003; 167: 211-277.

6 Park JH, Jegal Y, Shim TS, et al. Hypoxemia and arrhythmia during daily activities and six-minute walk test in fibrotic interstitial lung diseases. J Korean Med Sci 2011; 26: 372-378.

$7 \quad$ Nitzan M, Romem A, Koppel R. Pulse oximetry: fundamentals and technology update. Med Devices (Auckl) 2014; 7: 231-239.

8 Ortega R, Hansen CJ, Elterman K, et al. Videos in clinical medicine. Pulse oximetry. N Engl J Med 2011; 364 : e33.

9 Batchelder PB, Raley DM. Maximizing the laboratory setting for testing devices and understanding statistical output in pulse oximetry. Anesth Analg 2007; 105: Suppl., S85-S94.

10 Spicknall KE, Zirwas MJ, English JCIII. Clubbing: an update on diagnosis, differential diagnosis, pathophysiology, and clinical relevance. J Am Acad Dermatol 2005; 52: 1020-1028.

11 Van Ginderdeuren F, Van Cauwelaert K, Malfroot A. Influence of digital clubbing on oxygen saturation measurements by pulse-oximetry in cystic fibrosis patients. J Cyst Fibros 2006; 5: 125-128.

\section{Guidance for the regulatory status of allergen extracts in clinical trials}

To the Editor:

Following the introduction of the guidelines on clinical development and regulation of marketing authorisation for allergen extracts [1], there is an ongoing discussion on their regulatory status (i.e. authorised or unauthorised (off label)) when applied in interventional or observational clinical trials [2]. Since in most European Union (EU) countries, many allergen extracts either do not have a marketing authorisation or are not authorised for the intended application within a study protocol, it is often unclear which documents are needed for submission to an Independent Ethics Committee (IEC) and the Competent Authority.

Within clinical interventional or observational trials, allergen extracts can have different applications, i.e. as diagnostic tools (e.g. skin prick tests), as test or comparator products, as (standard) therapy, as challenge agents (e.g. nasal and inhalation provocation tests, inducing an immunological/physiological response) or as outcome measures. Depending on the application within a clinical protocol, allergen extracts can thus have different regulatory status requiring different product documentation (table 1) [3]. We present an overview of how to facilitate documentation for IEC and Competent Authority submission when an allergen extract is part of a clinical study.

Noninvestigational medicinal products (NIMPs) include agents other than the test product, the comparator product or placebo (the so-called investigational medicinal products (IMPs), and may be 
TABLE 1 Regulatory status of allergen extracts in clinical interventional research

$\begin{array}{lccc}\text { Application of allergen extract } & \begin{array}{c}\text { Regulatory status } \\ \text { in a clinical study }\end{array} & \begin{array}{r}\text { Regulatory documents } \\ \text { required for IEC and CA }\end{array}\end{array}$

\section{Interventional tests}

Skin prick test (diagnostic)

Skin prick test loutcome measure)

Provocation test (nasal/bronchial)

\begin{abstract}
NIMP (yes) NIMP (yes) NIMP (yes) NIMP (no)
\end{abstract}

$$
\begin{aligned}
& \text { IMP (no) } \\
& \text { IMP (yes) } \\
& \text { IMP (no) } \\
& \text { NIMP (yes) } \\
& \text { NIMP (yes) }
\end{aligned}
$$

$\begin{array}{cc}\text { SmPC } & \text { No } \\ \text { SmPC } & \text { No } \\ \text { SmPC } & \text { No } \\ \text { GMP certificate } & \text { No } \\ \text { manufacturer's product description } & \\ \text { IMPD } & \text { Yes } \\ \text { SmPC } & \text { No } \\ \text { IMPD } & \text { Yes } \\ \text { SmPC } & \text { No } \\ \text { SmPC } & \text { No }\end{array}$

IEC: Independent Ethics Committee; CA: Competent Authority; NIMP: noninvestigational medicinal product; IMP: investigational medicinal product; IMPD: Investigational Medicinal Product Dossier; SmPC: Summary of Product Characteristics; GMP: Good Manufacturing Practice.

provided to subjects participating in a study. NIMPs comprise agents used for preventive, diagnostic or therapeutic indications, and to induce a physiological response in a clinical study [4]. Both NIMPs and IMPs can be either authorised or unauthorised in one or more EU Member States. When applied in an interventional (observational) clinical study, documentation on both NIMPS and IMPs should be submitted to an IEC and Competent Authority in line with their marketing authorisation status within the country (or countries) where the study protocol is submitted.

In June 2016, the new Clinical Trial Regulation EU number 536/2014 will be issued for all clinical studies performed within the EU [5]. In this regulation, the term "NIMP" will be changed into "Auxiliary Medicinal Product", indicating a medicinal product, other than an IMP, used in a clinical trial in line with the study protocol. Documentation to be provided to the IEC and Competent Authority depends on the actual marketing authorisation status for the intended application of the product within the study.

In interventional clinical trials, it is strongly recommended that NIMPs with a marketing authorisation within the participating EU Member State are used. If not, the next choice should include NIMPs with a marketing authorisation within another EU Member State or, next, NIMPs with a marketing authorisation in an ICH (International Conference on Harmonisation of Technical Requirements for Registration of Pharmaceuticals for Human Use) country or a third country having a mutual recognition agreement with the EU (MRA country). If this is not possible, the next choice should be NIMPs with a marketing authorisation in another third country. Alternatively, a NIMP with no marketing authorisation, like most allergen extracts, may be used provided an adequate justification is included in the study protocol. Additionally, if a NIMP is unauthorised or if a NIMP is modified while such modification is not covered by a marketing authorisation, it should be ensured that the product has been manufactured according to EU GMP (Good Manufacturing Practice) by the providing industry or pharmacy, referring to Article 63(1) or to at least an equivalent standard, in order to ensure adequate product quality [6].

Allergen extracts aimed for NIMP application within a clinical study protocol often have no marketing authorisation in EU countries. Nevertheless, their use can be permitted in clinical trials if an adequate justification has been provided and approved by an IEC or Competent Authority.

0 @ERSpublications

Allergen extracts for NIMP use without EU marketing authorisation can be used if adequate justification is provided http://ow.ly/Q8LdZ

Zuzana Diamant ${ }^{1,2}$, Maurits van Maaren ${ }^{3}$, Roy Gerth van Wijk ${ }^{3}$, Jacobus R.B.J. Brouwers ${ }^{4}$ and Herman Pieterse ${ }^{5}$ ${ }^{1}$ Dept of Respiratory Medicine and Allergology, Institute for Clinical Science, Skane University Hospital, Lund, Sweden. ${ }^{2}$ Skane University, Department of Respiratory Medicine and Allergology Klinikgatan 18, S-221 81, Lund, Sweden. ${ }^{3}$ Dept of Allergology, Erasmus Medical Centre, Rotterdam, The Netherlands. ${ }^{4}$ Dept of Pharmacotherapy and Patient Care, University of Groningen, Groningen, The Netherlands. ${ }^{5}$ University of Ghent, Heymans Institute for Pharmacology, Ghent, Belgium.

Correspondence: Skane University, Department of Respiratory Medicine and Allergology Klinikgatan 18, S-221 81, Lund, Sweden. E-mail: z.diamant@gems.demon.nl 
Received: May 102015 | Accepted: May 142015

Conflict of interest: Disclosures can be found alongside the online version of this article at erj.ersjournals.com

\section{References}

1 Kaul S, May S, Lüttkopf D, et al. Regulatory environment for allergen-specific immunotherapy. Allergy 2011; 66: 753-764.

2 Zuberbier T, Werfel T. Is European legislation killing allergy diagnostics? Curr Opin Allergy Clin Immunol 2012; 12 : 475-476.

3 European Parliament, Council of the European Union. Directive 2001/20/EC of the European Parliament and of the Council of 4 April 2001 on the approximation of the laws, regulations and administrative provisions of the Member States relating to the implementation of good clinical practice in the conduct of clinical trials on medicinal products for human use. Off J Eur Commun 2001; L121: 34-44.

4 Health and Consumers Directorate-General. The rules governing medicinal products in the European Union: Volume 10 - guidance documents applying to clinical trials: guidance on investigational medicinal products (IMPs) and "noninvestigational medicinal products" (NIMPs) (rev. 1, March 2011). Brussels, European Commission, 2011.

5 European Parliament, Council of the European Union. Regulation (EU) No 536/2014 of the European Parliament and of the Council of 16 April 2014 on clinical trials on medicinal products for human use, and repealing Directive 2001/20/EC (1). Off J Eur Union 2014; L158: 1-76.

6 European Parliament, Council of the European Union. Directive 2001/83/EC of the European Parliament and of the Council of 6 November 2001 on the Community code relating to medicinal products for human use. Off J Eur Commun 2001; L311: 67-128.

Eur Respir J 2015; 46: 1223-1225 | DOI: 10.1183/13993003.00729-2015 | Copyright CERS 2015 